\title{
Ocular elongation and retraction in foveated reptiles
}

Ashley M. Rasys ${ }^{1}$, Shana H. Pau², Katherine E. Irwin ${ }^{1}$, Sherry Luo², Paul A. Trainor ${ }^{3,4}$, Douglas B. Menke ${ }^{2}$, and James D. Lauderdale ${ }^{1,5}$

${ }^{1}$ Department of Cellular Biology, The University of Georgia, Athens, GA 30602, USA; ${ }^{2}$ Department of Genetics, The University of Georgia, Athens, GA 30602, USA; ${ }^{3}$ Stowers Institute for Medical Research, Kansas City, MO 64110 USA; ${ }^{4}$ Department of Anatomy \& Cell Biology, The University of Kansas School of Medicine, Kansas City, KS 66160; ${ }^{5}$ Neuroscience Division of the Biomedical and Health Sciences Institute, The University of Georgia, Athens, GA 30602, USA

Running title: Eye morphogenesis in foveated lizards

Key words: Anole, chameleon, lizard, eye, development, fovea, ocular morphogenesis, myopia

Corresponding author and person to whom request should be addressed:

James D. Lauderdale

Department of Cellular Biology

University of Georgia

22 Athens, GA 30602, USA

23 Ph. 706-542-7433

24 Fax: 706-542-4271

e-mail: idlauder@uga.edu

Douglas B. Menke

28 Department of Genetics

29 University of Georgia

30 Athens, GA 30602, USA

31 Ph. 706-542-9557

32 Fax: 706-542-3910

33 e-mail: dmenke@uga.edu

\section{Funding:}

This work was funded by National Science Foundation awards 1149453 to D.B.M. and 1827647 to D.B.M. and J.D.L. and a Society for Developmental Biology Emerging Models grant to A.M.R. A.M.R. was supported by NIH training grant T32GM007103 and by an ARCS Foundation Scholarship. P.A.T is supported by the Stowers Institute for

Disclosure Statement: The authors have nothing to disclose. The funders of this research had no role in study design, data collection and analysis, decision to publish, or preparation of the manuscript. 
Rasys et al.

Eye morphogenesis in foveated lizards

\section{Abstract}

2 Background: Pronounced asymmetric changes in ocular globe size during eye

3 development have been observed in a number of species ranging from humans to

4 lizards. In contrast, largely symmetric changes in globe size have been described for

5 other species like rodents. We propose that asymmetric changes in the three-

6 dimensional structure of the developing eye correlate with the types of retinal

7 remodeling needed to produce areas of high photoreceptor density. To test this idea, we

8 systematically examined three-dimensional aspects of globe size as a function of eye

9 development in the bifoveated brown anole, Anolis sagrei.

11 Results: During embryonic development, the anole eye undergoes dynamic changes in

12 ocular shape. Initially spherical, the eye elongates in the presumptive foveal regions of

13 the retina and then proceeds through a period of retraction that returns the eye to its

14 spherical shape. During this period of retraction, pit formation and photoreceptor cell

15 packing are observed. We found a similar pattern of elongation and retraction

16 associated with the single fovea of the veiled chameleon, Chamaeleo calyptratus.

18 Conclusions: These results, together with those reported for other foveated species, 19 support the idea that areas of high photoreceptor packing occur in regions where the 20 ocular globe asymmetrically elongates and retracts during development.

\section{Key Findings}

- The eyes of the brown anole, Anolis sagrei, and veiled chameleon, Chamaeleo calyptratus undergo dynamic asymmetrical changes in ocular shape during development.

- In both species, asymmetric elongation and retraction of the ocular globe is associated with fovea morphogenesis.

- Pit formation and photoreceptor cell packing in the foveal area occur when the corresponding region of the ocular globe is retracting relative to adjacent regions. 
Rasys et al.

Eye morphogenesis in foveated lizards

\section{Introduction}

2 Decades of experimental work have revealed a great deal about the developmental mechanisms that govern the patterning, differentiation, and growth of the vertebrate eye. Much of this understanding has come from functional studies in mouse, rat, chicken, Xenopus, zebrafish, and medaka. ${ }^{1,2}$ Additional insights have come through genetic studies of human syndromes that feature eye defects. ${ }^{3}$ Consequently, we know a great deal about the genes and signaling pathways that regulate development of the core structures common to all vertebrate eyes, including the cornea, lens and retina. ${ }^{4-7}$ Missing from our current understanding of vertebrate eye development, however, is detailed knowledge about the developmental pathways that regulate the formation of specialized structures that are only present in the eyes of certain vertebrate species. These structures include the conus papillaris, areas or streaks, and foveae. Studies of eye formation in diverse vertebrate groups are needed to determine how these specialized structures form and to achieve a more complete understanding of vertebrate eye development and evolution. Notably, modern investigations of eye development have almost completely excluded reptiles, a tremendously successful amniote group represented by over 10,000 extant species. ${ }^{8}$

Although eye development in reptiles remains poorly studied, other aspects of reptilian biology have been actively explored. For instance, Anolis, a lizard genus with approximately 400 recognized species, has served as an important model system for studies of evolution, ecology, physiology, behavior, and neuroendocrinology for many years. ${ }^{9}$ More recently, Anolis has also emerged as a system to investigate reptile development and the mechanisms that contribute to morphological evolution. ${ }^{10-16}$ The

24 brown anole lizard, Anolis sagrei, is particularly well-suited for developmental studies 25 due its small size, ease of husbandry, continuous egg production, high fertility, and low cost. In addition, ex ovo culture systems and gene-editing have been established for

27 this species, which presents the opportunity for pharmacological and genetic

28 manipulation of Anolis embryos during development. 12,17,18 Of interest for studies of eye

29 development, Anolis lizards possess specialized structures that include a bifoveated 30 retina and a highly vascularized conus papillaris. ${ }^{19,20}$

\section{Page $\mathbf{3}$ of $\mathbf{2 6}$}


Rasys et al.

Eye morphogenesis in foveated lizards

Here we describe morphological and histological aspects of eye development in $A$. sagrei. We pay particular attention to alterations in ocular globe shape, which is an interesting, but poorly understood, aspect of eye development. Although typically only studied postnatally in the context of myopia in humans, ${ }^{21-23}$ changes in ocular shape during embryonic development have been observed in a number of foveated species, including humans, ${ }^{24-30}$ and non-human primates, ${ }^{26,31}$ as well as geckos, ${ }^{32,33}$ suggesting the presence of a conserved morphogenetic mechanism. The bifoveated brown anole is a good model system in which to study the mechanisms underlying fovea development in a vertebrate eye. In this study, we provide the first systematic three-dimensional assessment of the dynamic changes in ocular shape with an emphasis on ocular

11 elongation and retraction and its association with fovea formation.

Material and methods

\section{Animals}

All experimental procedures were conducted in accordance with the National Institutes of Health Guide for the Care and Use of Laboratory Animals under protocols approved and overseen by the University of Georgia (anoles) and Stowers Institute for Medical Research (chameleons) Institutional Animal Care and Use Committees. Anolis sagrei lizards were maintained in a breeding colony at the University of Georgia following guidelines described by Sanger et al., 2008. ${ }^{34}$ Eggs were collected weekly from natural matings and placed in $100 \times 15 \mathrm{~mm}$ lidded petri dishes containing moist vermiculite and incubated at $27-28^{\circ} \mathrm{C}$ and $70 \%$ humidity. Adults and hatchlings were euthanized using methods consistent with the American Veterinary Medical Association (AVMA) Guidelines for the Euthanasia of Animals. ${ }^{35,36}$ Chamaeleo calyptratus were maintained in a breeding colony at the Stowers Institute for Medical Research (Kansas City, Missouri) following guidelines described by Diaz et al., $2015^{37}$ and Diaz et al., $2019 .{ }^{38}$ Eggs were collected at the time of oviposition and incubated at $26-28^{\circ} \mathrm{C}$ and $50 \%$ humidity on damp vermiculite. Male and female embryos of both species were used for these studies. 
Rasys et al.

Eye morphogenesis in foveated lizards

\section{$1 \quad$ Staging}

2 Embryonic development of Anolis lizards typically takes place over a 30-33 day period, starting with fertilization, which takes place internally. ${ }^{39}$ Early embryogenesis

4 proceeds within the oviduct. $A$. sagrei embryos obtained from eggs that were collected 5 after egg-laying were staged as described by Sanger et al., 2008. ${ }^{39}$ Embryos younger pre-laying followed by a number. We describe here 5 PL timepoints, which includes the stage embryos were collected from gravid adult females following euthanasia. $C$. calyptratus embryos were staged following criteria described by Diaz et al., 2017 and

11 Diaz et al., 2019 and stage matched to the anole using Sanger's morphological criteria. ${ }^{38-40}$

\section{Dissection}

14 Lizard embryos were removed from their shells using a blunt pair of forceps and iris 15 scissors in 1x phosphate-buffered saline (PBS; $137 \mathrm{mM} \mathrm{NaCl}, 2.7 \mathrm{mM} \mathrm{KCl}, 10 \mathrm{mM}$ $16 \mathrm{Na}_{2} \mathrm{HPO}_{4}, 1.8 \mathrm{mM} \mathrm{KH}_{2} \mathrm{PO}_{4}, \mathrm{pH}$ 7.4). Upon removal of yolk and amniotic sac with fine 17 forceps, embryos were placed into $60 \mathrm{ml}$ of fresh $1 \times$ PBS solution with $1 \mathrm{ml}$ of $0.4 \%$ 18 pharmaceutical grade, neutrally buffered tricaine (TRICAINE-S; Western Chemical Inc)

19 to anesthetize for imaging. Eyes were enucleated from embryo stages $>4$ with fine 20 forceps and placed in Bouin's fixative at $4^{\circ} \mathrm{C}$ overnight on a rocker. Following fixation, 21 eyes were washed five times at 15 min per wash in 1x PBS. Specimens were stored in

$2270 \%$ ethanol solution $(\mathrm{EtOH})$ at $4^{\circ} \mathrm{C}$ until processed for histology. Whole chameleon

23 embryos were dissected from eggs in a similar manner, fixed in Bouin's at $4^{\circ} \mathrm{C}$

24 overnight, washed in 1x PBS, and stored in $70 \% \mathrm{EtOH}$ prior to shipment from the

25 Stowers Institute for Medical Research. Upon arrival at the University of Georgia, 26 embryos were slowly rehydrated in a series of graded EtOH/PBS solutions. Once fully 27 rehydrated, eyes were carefully removed from embryos.

\section{Whole Eye Measurements}

Prior to fixing, anole eyes were positioned in both a lateral and dorsal orientation and imaged with a ZEISS Discovery V12 SteREO microscope and AxioCam while in 1x PBS 
Rasys et al.

Eye morphogenesis in foveated lizards

solution. AxioVision 4 software (Carl Zeiss Microlmaging) was used to take axial measurements along the dorsoventral $(y)$, nasotemporal $(x)$, and lateromedial $(z)$ aspects of imaged eyes from embryos at Sanger stages 4-18, the hatchling (Hch), and the adult (Adt). The lateral view, which encompassed the whole cornea, was used to measure the $y$ - and $x$-axes, whereas the dorsal view was imaged to obtain $x$ - and $z$ axes. Because $x$-axial measurements can be acquired from both views, we included only the dorsal $x$-axial value in our dataset and used the other value as a control for proper orientation. Measurements from eyes that were not correctly positioned were excluded from the dataset. We normalized the $x$ - and $z$-axial measurements from each lizard to that same individual's $y$-axis and then multiplied this number to the mean $(\mu)$ of

11 that individual's stage group $y$-axis $\left(\mu_{\mathrm{y}}\right), \frac{\mathrm{x} \text { or } \mathrm{z}}{\mathrm{y}} * \mu_{\mathrm{y}}=\mathrm{x}$ - or Z-axis $\mathrm{N}$. To identify trends in

12 ocular growth, a ratio was then calculated for each axis by taking the raw y-axis dataset 13 and the normalized datasets ( $x$ - and $z$-axis $\mathrm{N}_{\mathrm{N}}$ ) from every lizard and dividing these values 14 with the corresponding $\mu$ of the raw $y-, x-$, and $z$-axial lengths of the hatchling,

$15 \frac{\mathrm{y} \text {-axis, } \mathrm{x} \text {-axisN, or } \mathrm{z} \text {-axisN (St 4-18; Hch; Adt) }}{\mu \mathrm{y}, \mu \mathrm{x}, \text { or } \mu \mathrm{z}(\mathrm{Hch})}$. Chameleon eyes were processed as reported

16 above except imaging was performed post-fixation and rehydration. Prism 7 (GraphPad

17 Software) and JMP V14.1 (JMP SAS) were used for graph generation and data

18 analyses. As a few of the sample groups did not have a normal distribution, we used a

19 nonparametric one-way ANOVA (Kruskal-Wallis) and Mann-Whitney for our statistical

20 analyses.

\section{$21 \quad$ Paraffin Sectioning}

22 Eyes were dehydrated in a series of graded ethanol solutions $70 \%, 80 \%, 90 \%, 96 \%$

23 and $100 \%$ (twice) for a minimum of $15 \mathrm{~min}$ each and then soaked in xylene for a total of

$2430 \mathrm{~min}$ for all embryonic stages. Tissue specimens were incubated in a series of 3

25 paraffin wax jars for $30 \mathrm{~min}$ at $65^{\circ} \mathrm{C}$, embedded in paraffin, and serially sectioned

26 horizontally at $10 \mu \mathrm{m}$. In adult specimens, the dorsal aspect of the eye was punctured

27 with a $0.15 \mathrm{~mm}$ minutien pin prior to processing in xylenes and paraffin waxes to

28 facilitate wax entry. Processing time in xylene in the adult was extended up to a total of

$292 \mathrm{hrs}$. Eyes were serial sectioned on a horizontal plane. Sections were stained with

30 hematoxylin \& eosin following standard protocols and mounted in Cytoseal (Thermo 
Rasys et al.

Eye morphogenesis in foveated lizards

1 Scientific $^{\mathrm{TM}}$ Richard-Allan Scientific ${ }^{\mathrm{TM}}$ ). Photomosaic images were generated using a

2 KEYENCE BZ-700 microscope with Keyence image stitching software. Adobe

3 Photoshop CC (2017.01 release) was used to digitally enhance contrast and adjust

4 white balance of images.

\section{Results}

\section{Anatomy of the adult anole eye}

Laterally positioned in the skull, the adult eye externally is oblate spheroid in shape with a prominent convex cornea slightly biased toward the nasal region (Figure 1a,b). Peripheral to the cornea is the sclera sulcus, whose curvature is supported by 14 sclera ossicles (Figure 1b,c). The sclera ossicles are uniformly orientated in a patterned ring except for a slight extension in the temporal region of the eye (Figure 1c). The anole's radial pupil is positioned centrally, fashioned by a heavily pigmented iris with an array of iridophores and melanophores that extend into the circumferential sclera sulcus (Figure $1 \mathrm{~b}, \mathrm{c})$. The iris is asymmetric with dorsal and ventral notches defining the boundary between the larger temporal and smaller nasal region (Figure 1c, arrowheads). Dorsally, a protruding blood vessel is present that extends from the optic nerve, wraps around the region of the center fovea (a small bulge in the medial region), and dissipates towards the dorsal nasal area of the eye (Figure 1b, narrow arrowheads). The optic nerve (not shown) exits the eye ventrally and temporally to the central fovea.

Internally, the adult anole eye is very similar to other vertebrates, possessing a cornea, iris/ciliary body, lens, and retina (Figure 2a). Anteriorly, the transparent cornea,

23 which is exterior to the lens and iris, is composed of epithelial, stromal, and endothelial

24 layers (Figure 2a, Rasys et al., in prep). These layers thicken towards the limbal area 25 where the cornea and anterior margin of the sclera ossicles meet. The sclera ossicles extend from the limbal area outward towards the sclera proper in overlapping sheets

27 making up the sclera sulcus. Underlying this sclera sulcus is the long thin ciliary body

28 (Figure 2a). The ciliary body lacks a ciliary process and comprises an inner non-

29 pigmented layer and an outer pigmented epithelial layer (Rasys et al., in prep). These

30 layers extend from the neural retina to the iris-ciliary boundary. Beyond this boundary is

31 the iris proper which is closely associated with the lens (Figure 2a). Both the inner and 
Rasys et al.

Eye morphogenesis in foveated lizards

1 outer iris epithelium are pigmented. The lens is oval shaped with a central nucleus,

2 inner cortex, and outer annular pad (Figure 2a). Anchoring the lens are zonular fibers

3 that extend from the ciliary inner non-pigmented epithelium (Figure 2a). Posteriorly, the

4 eye includes the retina, retinal pigmented epithelium, choroidal, and sclera layers. The

5 neural retina is avascular and composed of a ganglion cell layer (GCL), inner and outer

6 plexiform layers (IPL and OPL), inner and outer nuclear layers (INL and ONL) (Figure

$72 b, c)$.

8 Each eye has two foveae with one in the central retina and a second in the temporal retina (Figure 2). The central fovea, located slightly temporal (4-5 degrees) to the optical axis (the central or mid-point of the eye), exhibits a distinct pit accompanied by a higher

11 density of photoreceptor cells compared to the peripheral retina, as has been previously

12 described for other anoles. ${ }^{19,20}$ The retina at the central foveola is devoid of the GCL,

13 INL and ONL (Figure 2a,c). The retina in the parafoveal region has a larger number of

14 cell bodies in the INL and ONL compared to the peripheral retina (Figure 2a,c). A

15 second, shallower fovea is located in the temporal retina roughly 45 degrees from the

16 optical axis or 40 degrees from the visual axis (central fovea) (Figure 2). This temporal

17 fovea exhibits a shallow pit accompanied by an increase in photoreceptor cell density.

18 At its center, all the retinal cell layers are retained, but layers are thinner than the

19 surrounding peripheral retina (Figure $2 b$ ). The temporal fovea is approximately one-third

20 the area of the central fovea, with fewer photoreceptor cells.

\section{Eye formation}

22 In anoles, fertilization takes place internally and embryonic development occurs over

23 a 30-33 day period. ${ }^{39}$ As a consequence, the earliest stages of eye development take

24 place while the eggs are in the oviduct. Embryonic stages pre-egg lay are denoted with

25 the prefix "PL". Stages post-egg lay use the Sanger staging series ${ }^{39}$ and are denoted

26 using the prefix "St". Stages PL 3-5 correspond to Sanger stages St 1-3. ${ }^{39}$ For these

27 earliest timepoints we use the PL nomenclature to help distinguish pre-lay stages (PL 1-

28 5) from post-lay stages (Sanger St 4-19).

29 The initial stages of eye development occur between PL 1 and PL 5. Optic vesicle

30 formation is evident in embryos at PL 1 (Figure 3a), which is about 1 day after

31 fertilization and 3 days before egg lay. The lens placode is visible at PL 2 (see 
Rasys et al.

Eye morphogenesis in foveated lizards

1 developmental poster in supplementary data). By PL 3, the optic cup and lens pit are

2 present (Figure 3b). Rapidly following this period, the cornea separates (PL 5) from the

3 newly minted lens vesicle (PL 4) and the optic fissure begins to close (PL 5)

4 (developmental poster). At the time of egg lay ( St 4$)$, the anole eye is spherical, trace

5 amounts of pigmentation are evident in the temporal region of the eye (Figure $3 c$ ) and

6 present nasally by St 5 (Figure 3d). At this time (St 5), the prospective central and

7 temporal foveal regions are evident as thickenings in the retina, also defined as retinal

8 mounding (Figure 3g, Rasys et. al., in prep). St 5 also marks the first appearance of the

9 sclera sulcus, evidenced by a slight depression in the temporal area, which extends to

10 the nasal side by St 6 (Figure 3d). Eventually, the sclera sulcus forms a complete ring

11 encircling the cornea (developmental poster, whole eye stages 6-8). Shortly following

12 this period, pigment begins to increase - initially in the iris between St 6-10 and then

13 throughout the rest of the eye. In the iris, pigment is deposited first as a narrow band

14 along the horizontal axis (St 6-7) before radiating outward throughout the dorsal and

15 ventral regions (St 8-9) (Poster, Whole eye). By St 10, pigment in the iris is black and

16 evenly distributed. Granules are also just becoming obvious throughout the whole eye,

17 but more so in the temporal region. At St 13 , retinal mounding is no longer present in

18 the central and reduced in the temporal foveal regions (Figure 3h). The eye is a light

19 brown color which darkens between St 15-17 and is completely black by the time of

20 hatching (Figure 4b; developmental poster). During this period the sclera ossicles that

21 shape the sulcus and provide support to the underlying ocular structures, are starting to

22 form. The sclera anlagen first manifests as a ring of pale conjunctiva papillae around the

23 cornea between St 11-12. By St 13, scleral sheets are present although they are small

24 and by St 15-16 these expand, radiating outward and eventually overlapping (St 17)

25 with the neighboring plates (developmental poster). Iridophores (reflective pigments)

26 scattered throughout the iris and sclera sulcus region, are also apparent during this

27 time.

\section{Eye morphogenesis}

29 The anole eye exhibits conspicuous asymmetric changes in morphology during 30 development (Fig 4). To assess potentially complex changes in the three-dimensional

31 shape of the globe, measurements were made along the three anatomical axes of the 
Rasys et al.

Eye morphogenesis in foveated lizards

1 eye at different stages of development. For this study, the dorsoventral axis was defined

2 as the $y$-axis, the nasotemporal axis was defined as the $x$-axis, and the lateromedial 3 axis was defined as the $z$-axis (Figure $4 a$ ). The lateromedial $(z)$ axis is also the optical

4 axis and passes through anterior (iris and lens) and posterior (retina) structures of the 5 eye.

At St 5 , the ocular globe is mostly spherical in shape (Figure $4 b$ ), with similar lengths along the three anatomical axes. At late mid-gestation (St 13-14), the ocular globe has a triangular appearance with increased lengths along the nasotemporal $(x)$ and lateromedial ( $\mathrm{z}$ ) axes compared to the length of the dorsoventral axis (Figure 3e,h; Figure $4 \mathrm{~b}$ ). Interestingly, whereas the nasal surface of the globe has a rounded appearance, both the temporal and medial surfaces have angular shapes. These differences in surface geometry suggest that elongation in the nasotemporal and lateromedial planes occurred largely by changes in the temporal and medial regions of the globe, respectively. The medial region of the globe corresponds to the area of the retina that gives rise to the central fovea, and the temporal region corresponds to that of the temporal fovea (Figure $3 g-i)$. The eye at this stage also exhibits considerably less pigmentation at the medial surface compared to the lateral surface (Figure 4b). At hatching, the ocular globe has a spherical shape with uniform pigmentation (Figure 3i; Figure $4 b)$. Remarkably, the globe at this stage has a smaller surface area than that of mid-gestational embryos (Figure 4b). The change in morphology and size of the globe in a hatchling compared to that of a mid-gestational embryo suggests that the eye undergoes asymmetric retraction in the areas encompassing the developing central and temporal foveae during the period between mid-gestation and hatching.

To better understand the dynamics of globe morphogenesis in the brown anole, axial measurements along the three anatomical planes were made of eyes at embryonic stages St 4-18, hatchlings (Hch), and adults (Adt) (Figure S1; Table S1). To facilitate a quantitative comparison of changes in the three-dimensional shape of the eye, the three measurements of each eye were converted to a standardized metric (see Whole Eye Measurements in Methods). Briefly, in this metric the axial length of the y-axis, which did not exhibit appreciable elongation and retraction during development, was used as a normalization factor for measurements along the $x$ - and z-axes. Variance due to

\section{Page 10 of 26}


Rasys et al.

Eye morphogenesis in foveated lizards

1 differences in embryo body and eye size within an individual's stage group was handled

2 by multiplying the normalized measurements for each individual by the group mean

3 value $(\mu)$ for the $y$-axis (Figure S1; Table S1). This operation resulted in an overall

4 reduction in variance seen at each stage in both $\mathrm{x}$ - and $\mathrm{Z}$-axis $\mathrm{N}_{\mathrm{N}}$ datasets, which

5 suggests that the degree to which an eye elongates and retracts is proportional to the embryo's eye size (Figure S2).

Using the hatchling eye as a reference, regional differences in globe morphogenesis as a function of developmental stage were assessed by taking the raw y-axis dataset and the normalized datasets ( $\mathrm{x}$ - and $\mathrm{z}$-axis $\mathrm{N}$ ) from every lizard and dividing these values with the corresponding mean of the raw $y-, x-$, and z-axial lengths of the hatchling (see

11 Whole Eye Measurements in Methods). This analysis revealed four distinct phases in ocular morphogenesis (Figure 4c).

13 Phase 1, which occurs between embryonic St 4-8, is characterized by rapid growth

14 of the eye. In embryos at St 4-5, the globe appears to expand uniformly along all 3 axes, which suggests that ocular growth during this period is equally distributed across the eye. Although the St 5 eye is $50 \%$ smaller than the hatchling eye, the eye at these

17 two stages is quite similar in overall shape (Figure 4b,c; Table S1). At St 6, the globe

18 begins to exhibit asymmetric expansion, with more growth along the nasotemporal and lateromedial axes compared the dorsoventral axis. By St 7, expansion along the lateromedial axis is greater than along the nasotemporal axis.

The start of the phase 2 is defined as the developmental timepoint when

22 morphological asymmetry of the globe is clearly visible. This condition is met at St 8 . It

23 is also at this point that the overall globe size is similar to that of the hatchling. Between

24 St 8 and the close of the second phase at St 14, the globe continues to expand

25 asymmetrically along the nasotemporal and lateromedial axes, with the more

26 pronounced expansion along the lateromedial axis (Figure 4c,d). The globe reaches

27 maximum lengths along the nasotemporal and lateromedial axes by St 14 . At this stage

28 the length of the lateromedial axis is $1.4 x$ that of the hatchling, and the length of the 29 nasotemporal axis is $1.2 x$ that of the hatchling (Figure $4 b, c)$.

$30 \quad$ Phase 3 is the epoch during which the globe begins to shorten in length along the 31 nasotemporal and lateromedial axes to regain a spherical shape. This phase 
Rasys et al.

Eye morphogenesis in foveated lizards

encompasses St 15-18 (Figure 4c,d), and corresponds to the time during which the

2 fovea acquires its distinctive morphological characteristics (Figure 3h,i). Upon completion of retraction, the eyes are slightly smaller than those of hatchlings (St 17-18;

4 Figure $4 \mathrm{c}, \mathrm{d}$ ). The fourth, and final, phase is characterized by a uniform expansion of the 5 globe, which begins at the close of St 18 and continues into adulthood (Figure 4c). From hatching, the eye doubles in size by the time the lizard reaches adulthood (Figure 4c).

To determine the magnitude of the asymmetric shape changes during ocular morphogenesis, measurements capturing the maximum extent of elongation (St 13-14, Figure 4c) were compared to those capturing the maximum extent of retraction (St 18Hch, Figure 4c). Axial measurements of globes from lizards at St 13, 14 and 18, and hatching were compared using one-way ANOVA analyses (nonparametric KruskalWallis test); normalized values were used for the nasotemporal and lateromedial axes (for mean and standard deviation, see Table. S1). Although measurements along the dorsoventral axis were not statistically different across these four developmental time periods ( $p$-value 0.1585 ; alpha $=0.05$ ), significant differences were observed for measurements along both the nasotemporal and lateromedial axes ( $p$-value $<0.0001$ for 17 both; alpha $=0.05$ ). (Figure 4d). Among the normalized nasotemporal (x-axis N datasets, St 13 and 14 measurements were significantly different from the St 18 and the hatchling ( $p$-value $<0.0001$ for both; alpha $=0.05$ ) but not between St 13 and 14 (p-value 0.1244). Similar results were observed among the normalized lateromedial (z-axis $)$ measurements. For St 18 compared to hatchling, $x$-axis $N$ and $z$-axis ${ }_{N}$ lengths were significantly different ( $p$ value $<0.0001$, and $p$-value of 0.0017 , respectively). This suggests that by St 18 ocular retraction is finished and the expansion phase is already well underway by the time of hatching. The large difference in mean $x$ - and $z-$ axis $_{N}$ lengths between St 14 and 18 (z-

27 axis 2647 to $1807 \mu \mathrm{m}$; x-axis, 2663 to $2151 \mu \mathrm{m}$ ) equated to a $32 \%$ and $19 \%$ reduction

28 in the central and temporal regions, respectively (Figure $4 d$ ).

Changes in intraocular pressure can be one mechanism that drives globe expansion and retraction. Using an inflated ball as a model, morphological indicators of a pressurized globe could include a taut ocular surface that resists deformation. 
Rasys et al.

Eye morphogenesis in foveated lizards

1 Conversely, using an under-inflated ball as a model, a previously-pressurized globe that 2 has lost pressure might exhibit wrinkles or folds of the surface and be more flaccid. The surfaces of globes prior to St 16 were smooth and taut to the touch. In contrast, starting at St 17, the exterior ocular surface was easily depressed with a pair of dull forceps and wrinkles could be clearly seen in about half of the eyes examined (7/13 St 17 lizards) (Figure 5). At St 18, wrinkling could still be detected (8/19 lizards), although it was not as pronounced. By hatching, the majority of globes (9/10 lizards) were completely smooth and once again the ocular surface was taut (Figure 5a). For developmental stages between St 14 and hatching, wrinkled eyes tended to have reduced normalized measurements along the lateromedial (z-axis $\mathrm{N}_{\mathrm{N}}$ ) compared to smooth eyes (Figure $5 \mathrm{c}$ ).

\section{Ocular elongation \& retraction in other foveated lizards}

To test if ocular elongation and retraction occurs in other foveated lizards, ocular morphogenesis was examined in the veiled chameleon lizard, Chamaeleo calyptratus. This species was chosen because it possesses a single, prominent central fovea that is completely devoid of all retina cell layers. ${ }^{41}$ Chameleon embryos were collected at several time points throughout development. To facilitate comparison with anoles, chameleon embryos were staged following criteria described by Diaz et al., 2017 and Diaz et al., 2019 and then matched to the anole using Sanger's morphological criteria. ${ }^{38-}$ ${ }^{40}$ Only embryonic stages were used for this study; chameleon embryos collected just prior to the expected hatching date are denoted as pre-hatch "pHch" (Table S2).

As observed for $A$. sagrei, ocular globe morphogenesis in $C$. calyptratus includes pronounced asymmetric elongation along the lateromedial axis (Figure 6). In contrast with $A$. sagrei, $C$. calyptratus embryos exhibit comparable expansion along both the dorsoventral and nasotemporal axes (Figure 6). As with the anole, ocular elongation is followed by a period of retraction that ends when the globe regains a spherical shape.

27 Compared with the anole, the elongated region in chameleon is less acute and occurs

28 over a broader area; in the anole, the elongated medial face appears more acute and

29 funnel-like (Compare St 13 chameleon in Figure 6B to St 13 anole in Figure 4b).

30 Despite this difference in morphology, the onset of ocular elongation and retraction

31 timing is nearly identical to anoles. For instance, ocular elongation begins around stage

\section{Page 13 of 26}


Rasys et al.

Eye morphogenesis in foveated lizards

16 and peaks by stage 14 . This is followed by ocular retraction, which starts at stage 15

2 and plateaus just before hatching (Figure 6c). The chameleon fovea also develops

3 during the period of retraction (between stages 16-18) (Figure 6c), and its progression

4 also matches that of the anole (data not shown).

\section{Discussion}

The work we present here is a first step in establishing the brown anole as a new model organism for fovea developmental studies. A primary motivation for choosing this lizard is that the anole eye contains two fovea that represent the extremes of fovea morphology: a central fovea with a pit devoid of all retina cell layers and a temporal

11 fovea with a shallow pit that retains the retinal layers. Most vertebrate species do not have fovea, but in those that do, the fovea can differ greatly in pit shape, depth, and diameter. ${ }^{19,42-45}$ In humans, only the GCL and INL retinal layers are laterally displaced,

14 while the ONL is retained. This results in a broad and relatively shallow foveal pit with a 15 high density of photoreceptor cells at its center. ${ }^{30,46-50}$ This is also true of most foveated non-human primates. Although in some species, the GCL and/or INL are retained, and as a result, only a rudimentary pit is present. ${ }^{45,51-54}$ The fovea of birds is variable, and, like the anole, some species are bifoveated, while others only possess a single fovea located either in the central or temporal retinal regions. ${ }^{44}$

Anoles are the only squamate genus known to have a bifoveated retina. Among the anole species studied, all have a prominent central fovea devoid of cell layers and a shallower temporal fovea that retains these layers. ${ }^{19,20,55}$ Slight variations in pit depth have been observed across anoles and correlate with prey size. ${ }^{19}$ For instance, anole species that routinely eat smaller prey have considerably deeper temporal foveae. As in

25 birds, the location of the fovea in different squamate reptiles varies. In diurnal geckos only a temporal fovea is present. ${ }^{56-60}$ Although all layers are generally present in these

27 lizards, the degree of lateral displacement ranges from partial to full layer retention. In

28 Lygodactylus, which is a gekkonid, and Gonatodes, which is a sphaerodacylid, the GCL 29 is nearly absent, while the INL is only thinned and ONL packing is present at the foveal 30 pit center. ${ }^{58}$ In contrast, Phelsuma geckos have only the shallowest of depressions 31 absent of any pronounced displacement of GCL and INL, resulting in a pit similar to the

\section{Page 14 of 26}


1 temporal fovea of anoles. ${ }^{58,59}$ In other lizards, including chameleons, the opposite is 2 generally true, with most having a large prominent central fovea devoid completely of 3 retinal cell layers. ${ }^{61,62}$

We observed four distinct phases of ocular morphogenesis in anoles and chameleons. The first was a period of symmetrical growth that occurred during the first week of embryonic development post-fertilization. At the end of this period, the retina was thicker and exhibited a mounded appearance in the prospective foveal regions (Figure $3 \mathrm{~g}$ ). This was followed by a second period, defined by asymmetrical growth, where the regions that eventually gave rise to the fovea became strikingly elongated. Coincidingly, this period also marks the gradually disappearance of retinal mounding

11 within the foveal regions (Rasys et. al., in prep). By late development, when the foveae

12 take on their characteristic morphology, these regions appear to undergo retraction

13 coincident with retinal remodeling, i.e., pit formation and photoreceptor cell packing. The

14 fourth phase was characterized by a uniform expansion of the globe. In both the brown

15 anole and the veiled chameleon the regions that undergo elongation and retraction are

16 localized to areas of the retina where the foveae develop. Additionally, these foveal

17 regions, characterized by early retinal mounding, undergo retina differentiation and

18 lamination prior to the rest of the retina (Rasys et. al., in prep). These observations

19 suggest a relationship between changes in retinal differentiation, ocular shape, and

20 foveal development.

21 We propose that ocular elongation followed by retraction are necessary steps in

22 the retinal remodeling needed to generate a fovea in vertebrates. Consistent with this

23 idea, evidence of asymmetrical globe development can be seen in the eyes of diurnal,

24 but not nocturnal, gecko species. Examples of this phenomenon can be seen in Figures

252 and 3 of the publication by Guerra-Fuentes and colleagues addressing the

26 embryology of the retinal pigmented epithelium in five species of sphaerodactyls, ${ }^{32}$ and

27 in Figures 3 and 4 of the publication by Griffing, Gamble and Bauer addressing pigment

28 development in nocturnal gekkonids (Gekko kuhli, Lepidodactylus lugubris) and diurnal

29 gekkonids (Phelsuma laticauda, Sphaerodactylus macrolepis). ${ }^{33}$ Among mammals,

30 asymmetric globe development has been observed only for foveated haplorrhine

31 primates but not for non-foveated primates or other mammalian species. ${ }^{24-31}$ Together,

\section{Page 15 of 26}


Rasys et al.

Eye morphogenesis in foveated lizards

1 these observations suggest that fovea morphogenesis is similar among foveated

2 vertebrates. It will be interesting to learn if the eyes of foveated birds and fish undergo

3 similar morphogenetic changes during development.

In the brown anole, we noted that the magnitude of asymmetric ocular shape changes during development appeared proportional to the extent of retinal remodeling associated with formation of the morphologically dramatic central fovea compared to the less distinct temporal fovea. We speculate that the process of elongation and retraction of required for fovea formation, and that the relative extent to which an eye elongates and retracts during fovea-genesis is directly proportional to the amount of retinal remodeling required to make that particular fovea. If true, this may explain the variability

11 of fovea morphology present within each foveated species.

Several different mechanisms could mediate changes in the size and shape of

13 the ocular globe during development. We hypothesize that changes in intraocular

14 pressure (IOP) contribute to asymmetric ocular morphogenesis in the brown anole. This

15 idea arises from our observations that the embryonic anole eye appears to be

16 pressurized during peak periods of elongation and deflated during retraction. The idea

17 that IOP can drive ocular growth is not a new one. Previous studies have shown that

18 IOP plays a pivotal role in regulating normal ocular growth in chick ${ }^{63}$ and increases in

19 IOP can lead to induced myopia (generalized axial elongation of the globe) in this

20 animal. ${ }^{64,65}$ In foveated primates, Hendrickson and Springer proposed a model where

21 high IOP induces pit formation due to inherent increased elasticity present at the foveal

22 avascular zone, while "retinal stretching" induced by ocular growth, facilitates the

23 centripetal movement of photoreceptor cells towards the foveal center ${ }^{66-68}$ Although, it

24 is possible that lack of blood vessels would predispose this region of the primate retina

25 to be more susceptible to IOP and, therefore, form a pit, this cannot explain pit

26 formation in the lizard. Anoles have a retina that is entirely avascular. ${ }^{43,55}$ This suggests

27 that if regional differences in retinal elasticity are present in the anole, they are unlikely

28 to be caused by avascular zones. Another challenge with this model, as applied to

29 anoles, is that it requires IOP to be high for pit formation to occur. In the brown anole, pit

30 formation occurs during the period that the eye is soft, indicating that IOP is low. 
Rasys et al.

Eye morphogenesis in foveated lizards

We propose, for anoles at least, that high IOP is involved in facilitating ocular

2 elongation, but that IOP is comparatively low during ocular retraction and pit formation.

3 As for Hendrickson and Springer's model, there must be additional mechanisms that

4 mediate regional differences in the elasticity of the foveal anlagen compared to other

5 regions of the ocular globe. We think it likely that regional and dynamic changes in the

6 elasticity of the tissues associated with the outer surface of the globe are required for

7 normal eye development in foveated lizards, and may be true for primates as well.

\section{Acknowledgements}

10 The authors thank Aaron Alcala, Sergio Minchey, Sukhada Samudra, Christina

11 Sabin, and Rebecca Ball of the Menke and Lauderdale research groups at the

12 University of Georgia, and Diana Baumann, Richard Kupronis, David Jewell, Alex

13 Muensch and Nikki Inlow of the Reptile and Aquatic Facility at the Stowers Institute for

14 Medical Research, for their help with animal husbandry, maintenance and care of the

15 anolis and chameleon colonies, respectively. The authors thank Drs. Jonathan

16 Eggenschwiler, Heike Kroeger and Robert Hufnagel, and Christina Sabin, Sukhada

17 Samudra, and Rida Osman for helpful discussions about this project and comments on

18 the manuscript. 


\section{References:}

2 1. Tsonis PA. Animal models in eye research. Academic Press; 2008.

3 2. Wittbrodt J, Shima A, Schartl M. Medaka--a model organism from the far East.

$4 \quad$ Nat Rev Genet. Jan 2002;3(1):53-64. https://doi.org10.1038/nrg704.

5 3. McKusick VA. Mendelian Inheritance in Man and its online version, OMIM. Am J

4. Miesfeld JB, Brown NL. Eye organogenesis: A hierarchical view of ocular

5. Chow RL, Lang RA. Early eye development in vertebrates. Annu Rev Cell Dev Biol. 2001;17:255-96.

6. Fuhrmann S. Eye morphogenesis and patterning of the optic vesicle. Curr Top Dev Biol. 2010;93:61-84. https://doi.org10.1016/b978-0-12-385044-7.00003-5.

$147 . \quad$ Saha MS, Servetnick M, Grainger RM. Vertebrate eye development. Curr Opin Genet Dev. 1992;2(4):582-8.

8. Uetz $P$, Stylianou A. The original descriptions of reptiles and their subspecies. Zootaxa. Jan 24 2018;4375(2):257-264.

https://doi.org10.11646/zootaxa.4375.2.5.

9. Losos JB, Schneider CJ. Anolis lizards. Curr Biol. Apr 28 2009;19(8):R316-8. https://doi.org10.1016/i.cub.2009.02.017.

21 10. Eckalbar WL, Lasku E, Infante CR, et al. Somitogenesis in the anole lizard and

11. Park S, Infante CR, Rivera-Davila LC, Menke DB. Conserved regulation of

12. Sanger TJ, Kircher BK. Model Clades Versus Model Species: Anolis Lizards as an Integrative Model of Anatomical Evolution. Methods Mol Biol. 2017;1650:285297. https://doi.org10.1007/978-1-4939-7216-6 19. 
Rasys et al.

Eye morphogenesis in foveated lizards

1 13. Sanger TJ, Revell LJ, Gibson-Brown JJ, Losos JB. Repeated modification of

16. Infante CR, Mihala AG, Park S, et al. Shared Enhancer Activity in the Limbs and

21. Chakraborty R, Read SA, Vincent SJ. Understanding myopia: Pathogenesis and 29

15. Tollis M, Hutchins ED, Stapley J, et al. Comparative Genomics Reveals Accelerated Evolution in Conserved Pathways during the Diversification of Anole Lizards. Genome Biol Evol. Feb 1 2018;10(2):489-506.

https://doi.org10.1093/gbe/evy013.

17. Infante CR, Rasys AM, Menke DB. Appendages and gene regulatory networks: Snakes. Dev Cell. Oct 12 2015;35(1):107-19.

https://doi.org10.1016/i.devcel.2015.09.003.

18. Rasys AM, Park S, Ball RE, Alcala AJ, Lauderdale JD, Menke DB. CRISPRCas9 Gene Editing in Lizards through Microinjection of Unfertilized Oocytes. Cell Reports. Aug 27 2019;28(9):2288-+. https://doi.org10.1016/j.celrep.2019.07.089.

19. Fite KV, Lister BC. Bifoveal vision in anolis lizards. Brain Behav Evol. 1981;19(34):144-54. https://doi.org10.1159/000121639.

20. Sannan NS, Shan X, Gregory-Evans K, Kusumi K, Gregory-Evans CY. Anolis carolinensis as a model to understand the molecular and cellular basis of foveal development. Exp Eye Res. Aug 2018;173:138-147. https://doi.org10.1016/j.exer.2018.05.012. mechanisms. Updates on Myopia. Springer, Singapore; 2020:65-94. 
Rasys et al.

Eye morphogenesis in foveated lizards

1 22. Harper AR, Summers JA. The dynamic sclera: extracellular matrix remodeling in

24. Badtke G. Entwicklungsmechanische Faktoren bei der Formgebung des

25. Ehlers N, Matthiessen ME, Andersen $\mathrm{H}$. The prenatal growth of the human eye.

26. Pilleri G. [Comparative anatomical examination of the "protuberantia scleralis" of the eye]. Acta Anat (Basel). 1960;41:131-7.

27. van L, Pilleri G. [Morphological research on the "scleral protuberance"]. Albrecht Von Graefes Arch Ophthalmol. 1961;163:1-9. https://doi.org10.1007/bf00684908.

28. Streeten BW. Development of the Human Retinal Pigment Epithelium and the Posterior Segment. Archives of Ophthalmology. Mar 01 1969;81(3):383-394.

29. Sondermann R. Die Bedeutung der Vererbung für die Entwicklung der Myopie. Albrecht von Graefes Archiv für klinische und experimentelle Ophthalmologie. 1950;151(1):200-208.

30. Hendrickson A. Development of Retinal Layers in Prenatal Human Retina.

31. Springer $A D$, Hendrickson $a E$. Development of the primate area of high acuity, 3 : 2015;166:29-35. temporal relationships between pit formation, retinal elongation and cone packing. Visual Neuroscience. Mar 2005;22(2):171-185. https://doi.org10.1017/S095252380522206X.

32. Guerra-Fuentes RA, Bauer AM, Daza JD. The embryology of the retinal pigmented epithelium in dwarf geckos (Gekkota: Sphaerodactylinae): a unique developmental pattern. BMC Developmental Biology. 2014;14(1):29. https://doi.org10.1186/1471-213X-14-29.

33. Griffing AH, Gamble T, Bauer AM. Distinct patterns of pigment development underlie convergent hyperpigmentation between nocturnal and diurnal geckos 
Rasys et al.

Eye morphogenesis in foveated lizards

34. SANGER TJ, HIME PM, JOHNSON MA, DIANI J, LOSOS JB. Laboratory

35. Association AVM. AVMA Guidelines for the Euthanasia of Animals: 2020 Edition.

36. Conroy CJ, Papenfuss T, Parker J, Hahn NE. Use of tricaine methanesulfonate

37. Diaz RE, Jr., Anderson CV, Baumann DP, et al. Captive Care, Raising, and

39. Sanger TJ, Losos JB, Gibson-Brown JJ. A developmental staging series for the lizard genus Anolis: a new system for the integration of evolution, development, and ecology. J Morphol. Feb 2008;269(2):129-37.

https://doi.org10.1002/jmor.10563.

38. Diaz RE, Jr., Shylo NA, Roellig D, Bronner M, Trainor PA. Filling in the phylogenetic gaps: Induction, migration, and differentiation of neural crest cells in a squamate reptile, the veiled chameleon (Chamaeleo calyptratus). Dev Dyn. Aug 2019;248(8):709-727. https://doi.org10.1002/dvdy.38.

Diaz RE, Jr., Bertocchini F, Trainor PA. Lifting the Veil on Reptile Embryology: The Veiled Chameleon (Chamaeleo calyptratus) as a Model System to Study Reptilian Development. Methods Mol Biol. 2017;1650:269-284. https://doi.org10.1007/978-1-4939-7216-6 18.

41. Hulke JW. On the chameleon's retina; a further contribution to the minute anatomy of the retina of reptiles. Philosophical Transactions of the Royal Society of London. 1866;(156):223-229.

42. Slonaker JR. A comparative study of the area of acute vision in vertebrates. Boston: Ginn \& company; 1897:1 p.l., p. [445]-502.

43. Walls GL, Walls GL. The vertebrate Eye and Its Adaptive Radiation. 1942:1-785. 
Rasys et al.

Eye morphogenesis in foveated lizards

1 44. Fite KV, Rosenfield-Wessels S. A comparative study of deep avian foveas. Brain,

45. Ross CF. The Tarsier Fovea: Functionless Vestige or Nocturnal Adaptation? In:

46. Hendrickson A. A morphological comparison of foveal development in man and

47. monkey. Eye (Lond). 1992;6 ( Pt 2):136-44. https://doi.org10.1038/eye.1992.29.

48. Hendrickson A, Possin D, Vajzovic L, Toth CA. Histologic development of the human fovea from midgestation to maturity. Am J Ophthalmol. Nov 2012;154(5):767-778 e2. https://doi.org10.1016/j.ajo.2012.05.007.

49. Hendrickson AE, Yuodelis $\mathrm{C}$. The morphological development of the human

50. Yuodelis $\mathrm{C}$, Hendrickson A. A qualitative and quantitative analysis of the human

51. fovea during development. Vision Res. 1986;26(6):847-55.

52. Stone J, Stone J, Johnston E, Johnston E. The topography of primate retina: a

53. DeBruyn EJ, Wise VL, Casagrande VA. The size and topographic arrangement

54. Collins $\mathrm{CE}$, Hendrickson $\mathrm{A}$, Kaas $\mathrm{JH}$. Overview of the visual system of tarsius. study of the human, bushbaby, and new- and old-world monkeys. [Case Report]. Journal of Comparative Neurology. Feb 20 1981;196(2):205-223. https://doi.org10.1002/cne.901960204. of retinal ganglion cells in the galago. Vision Res. 1980;20(4):315-27. https://doi.org10.1016/0042-6989(80)90018-8. The Anatomical Record Part A: Discoveries in Molecular, Cellular, and Evolutionary Biology. 2005;287A(1):1013-1025. https://doi.org10.1002/ar.a.20263. 
Rasys et al.

Eye morphogenesis in foveated lizards

1 55. Makaretz M, Levine RL. A light microscopic study of the bifoveate retina in the 2 lizard Anolis carolinensis general observation and convergence ratios. Vision

4 56. Underwood G. Reptilian retinas. Nature. Feb 3 1951;167(4240):183-5.

5 57. Underwood G. On the classification and evolution of geckos. Proceedings of the

58. Röll B. Gecko vision-retinal organization, foveae and implications for binocular

59. Tansley K. The retina of a diurnal gecko. Pflüger's Archiv für die gesamte

Physiologie des Menschen und der Tiere. 1961/05/01 1961;272(3):262-269. https://doi.org10.1007/BF00363014.

14 60. Tansley K. The gecko retina. Vision Res. 1964;4:33-37.

15 61. Hulke JW. On the Chameleon's Retina; A Further Contribution to the Minute

62. Detwiler SR, Laurens H. STUDIES ON THE RETINA THE STRUCTURE OF

63. Schmid KL, Hills T, Abbott M, Humphries M, Pyne K, Wildsoet CF. Relationship THE RETINA OF PHRYNOSOMA CORNUTUM. J Comp Neurol. Anatomy of the Retina of Reptiles. Philosophical Transactions of the Royal Society of London. 1866;156:223-229. 1920;32(3):347-356. between intraocular pressure and eye growth in chick. Ophthalmic and Physiological Optics. Jan 2003;23(1):25-33. https://doi.org10.1046/j.14751313.2003.00085.x.

64. Phillips JR, McBrien NA. Pressure-induced changes in axial eye length of chick and tree shrew: significance of myofibroblasts in the sclera. Investigative Opthalmology \&amp; Visual Science. Mar 2004;45(3):758-763. https://doi.org10.1167/iovs.03-0732.

65. Genest R, Chandrashekar N, Irving E. The effect of intraocular pressure on chick eye geometry and its application to myopia. Acta of Bioengineering and Biomechanics. 2012;14(2):3-8. https://doi.org10.5277/abb120201. 
Rasys et al.

1 66. Springer AD. New role for the primate fovea : A retinal excavation determines photoreceptor deployment and shape. 1999;16(0):629-636.

3 67. Springer AD, Hendrickson aE. Development of the primate area of high acuity. 1.

68. Provis JM, Dubis AM, Maddess T, Carroll J. Adaptation of the central retina for high acuity vision: cones, the fovea and the avascular zone. Prog Retin Eye Res. 
Rasys et al.

Eye morphogenesis in foveated lizards

\section{Figure Legends}

Figure 1. Adult anole eye. Top panel (a) shows adult male lizard with an enlarged view of its left eye. Bottom panels show a lateral view (b) and dorsal view (c) of a right eye. Directionality is designated by arrows and the letters $\mathrm{T}$ - temporal, $\mathrm{N}$ - nasal, $\mathrm{D}$ dorsal, and V - ventral. Markers indicate: white arrow heads with notches - dorsal (green) and ventral (white) iris notches; asterisks (magenta)- individual sclera ossicle sheets; narrow white arrow heads (b) - dorsal blood vessel, (c) temporal sclera ossicle deformation; black arrow - optic nerve; crosshair - dorsal optical axis; and scale bars $1 \mathrm{~mm}$.

Figure 2. Anole eye organization and retinal layout. Left panel (a) shows a diagram labeling structures present in a horizontally sectioned adult eye. Right panels show magnified views of temporal (b) and center (c) foveae. Note: temporal fovea shown in (b) is $150 \mu \mathrm{m}$ deep to the plane of section in (a). Markers indicate $\mathrm{GCL}$ - ganglion cell layer; INL - inner nuclear layer; ONL - outer nuclear layer; RPE - retina pigmented epithelium; Ch - choroid; Scl - sclera; and scale bars - $1 \mathrm{~mm} \mathrm{(a)} \mathrm{and} \mathrm{50 \mu m} \mathrm{(b} \mathrm{and} \mathrm{c).}$

Figure 3. Developmental series of early, mid, and late embryonic stages of eye morphogenesis. Top panel shows an array of early-stage embryos (PL1, PL3, and St 4) during optic vesicle (a), len's pit (b), and lens separation (c). Bottom panel shows later stages (St 5, 13, and Hch) along with histological sections through the eye's center horizontal plane. Black arrow - temporal eye region; open arrows - center and closed arrows - temporal retinal and fovea regions; and scale bars are $250 \mu \mathrm{m}(\mathrm{a}-\mathrm{c}, \mathrm{g}-\mathrm{i})$ and 1 $\mathrm{mm}(\mathrm{d}-\mathrm{f})$.

Figure 4. Anole eyes undergo asymmetrical ocular elongation followed by retraction during embryonic development. (a) Diagram illustrating orientation of $y$ - (green), $x$ (blue), and z-axis (magenta) ocular measurements. (b) Lateral (top) and dorsal (bottom) views of whole right eyes from early (St 5 ), mid (St 13), and late (Hch) stages. Ocular elongation and retraction phases are evident in the stage 13 embryo and hatchling, respectively. All eyes are to scale with one another; scale bar is $1 \mathrm{~mm}$. (c) Graph 
Rasys et al.

Eye morphogenesis in foveated lizards

1 displaying trends in ocular morphogenesis throughout development (St 4-Hch) and 2 adulthood (Adt F - adult female; Adt $\mathrm{M}$ - adult male). This graph was generated by

3 taking each lizard's ocular dimensions ( $y$-axis and normalized $x$ - and $z$-axis $)$ and

4 dividing it by the mean $(\mu)$ hatchling ocular dimensions ( $y-, x-$, and $z$-axis) to calculate a

5 ratio. (d) Direct comparison of ocular length along different axes reveals the degree of

6 ocular elongation (St 13-14) and subsequent retraction (St 18-Hch) in the anole eye.

8 Figure 5. The formation of surface wrinkles coincides with ocular retraction. (a) Dorsal views of whole right eyes from stages 16 - Hatchling showing the progressive steps of ocular retraction; scale bar $-1 \mathrm{~mm}$. (b) An enlarged view of the St 17 eye from panel

11 (a); arrow heads mark folds/wrinkles present along the outer ocular surface. (c) ocular

12 dimensions along the $z$-axes relative to the hatchling eye; eyes where folds/wrinkles

13 were observed are indicated in magenta.

15 Figure 6. Chameleon eyes also undergo asymmetrical ocular elongation followed by 16 retraction during embryonic development. Diagram (a) demonstrates how y- (green), x17 (blue), and z-axis (magenta) ocular measurements were made. Image (b) shows lateral 18 (top) and dorsal (bottom) views of whole fixed right eyes from early (St 6), mid (St 13), 19 and late (St 18) embryonic development. Like the anole, ocular elongation (St 13) and 20 retraction (St 18) phases are evident. All eyes are to scale; scale bar is $1 \mathrm{~mm}$. Graph

21 (c) summarizes trends in chameleon ocular morphogenesis throughout stages 4-pHch

22 (pHch - just prior to hatching). This graph was generated by taking each lizard's ocular 23 dimensions ( $y$-axis and normalized $x$ - and $z$-axis $N$ ) and dividing it by the mean $(\mu)$ pHch 24 ocular dimensions ( $y-, x-$, and z-axis) to calculate a ratio. Graph (d) compares the 25 degree of ocular elongation (St 14) and subsequent retraction (Hch and pHch) between 26 anoles (grey circles) and chameleons (color triangles: $y$-axis - green, $x$-axis $\mathrm{s}_{\mathrm{N}}$ - blue, 27 and $z$-axis $\mathrm{s}_{\mathrm{N}}$ - magenta). Calculations were made following same formula outlined above 28 in graph (c) for each respective species. Graph (e) indicates the relative percentage of 29 retraction occurring in chameleons between stage 14 and pHch. 
Figure 1
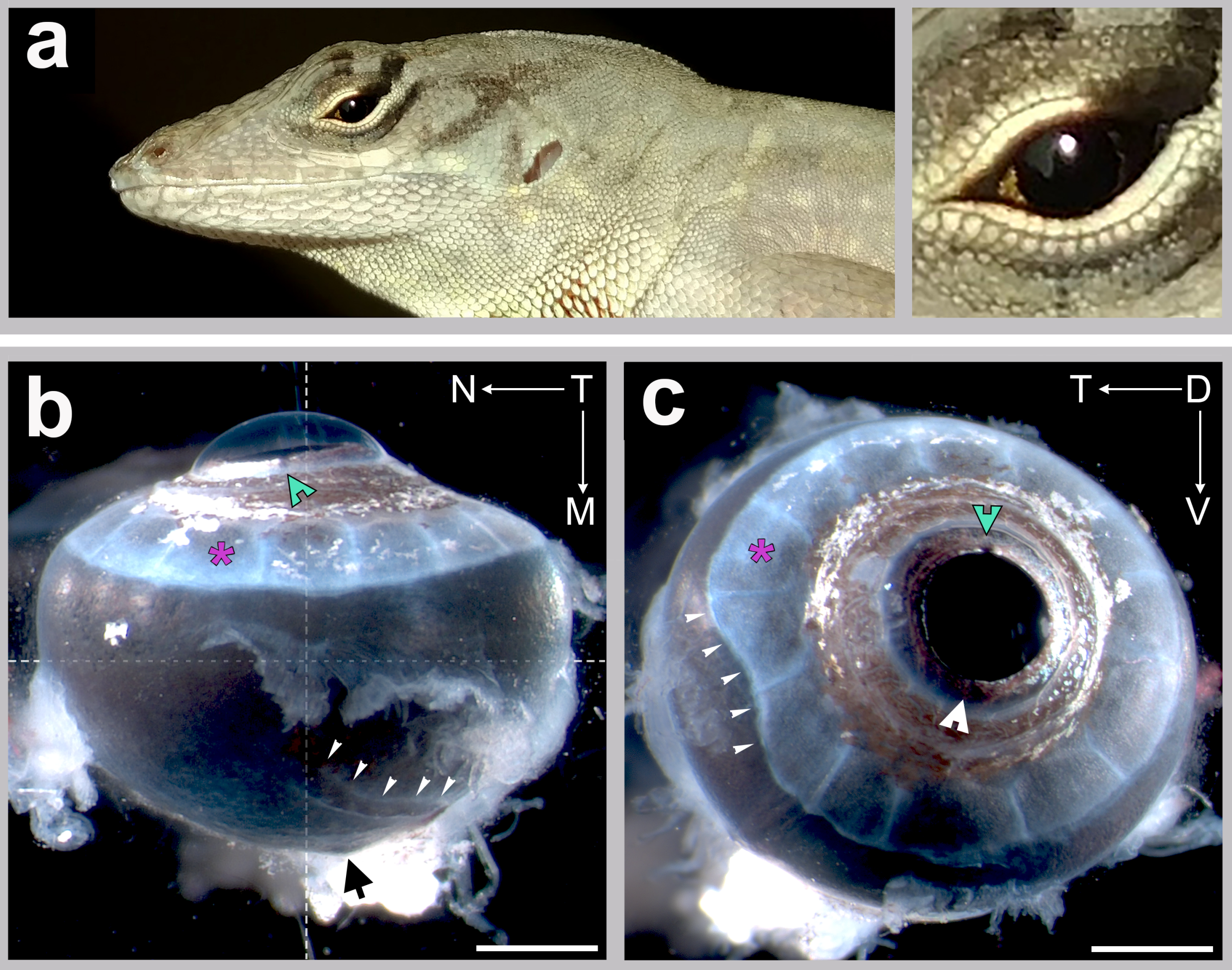

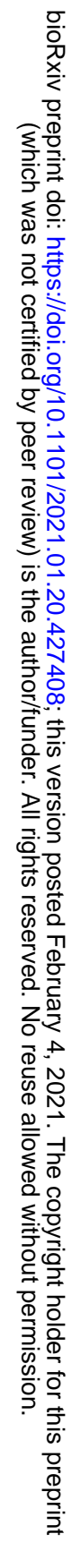

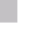



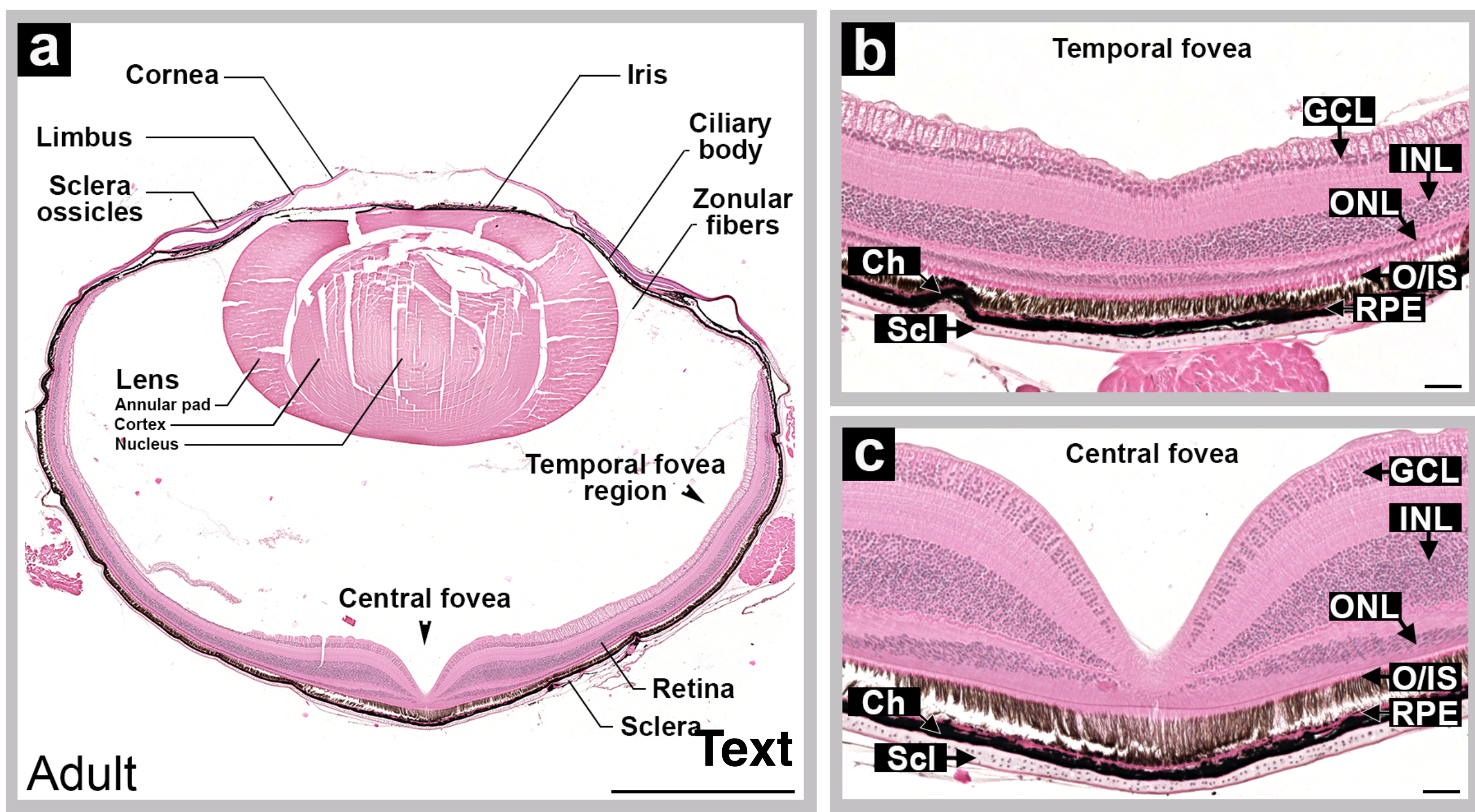

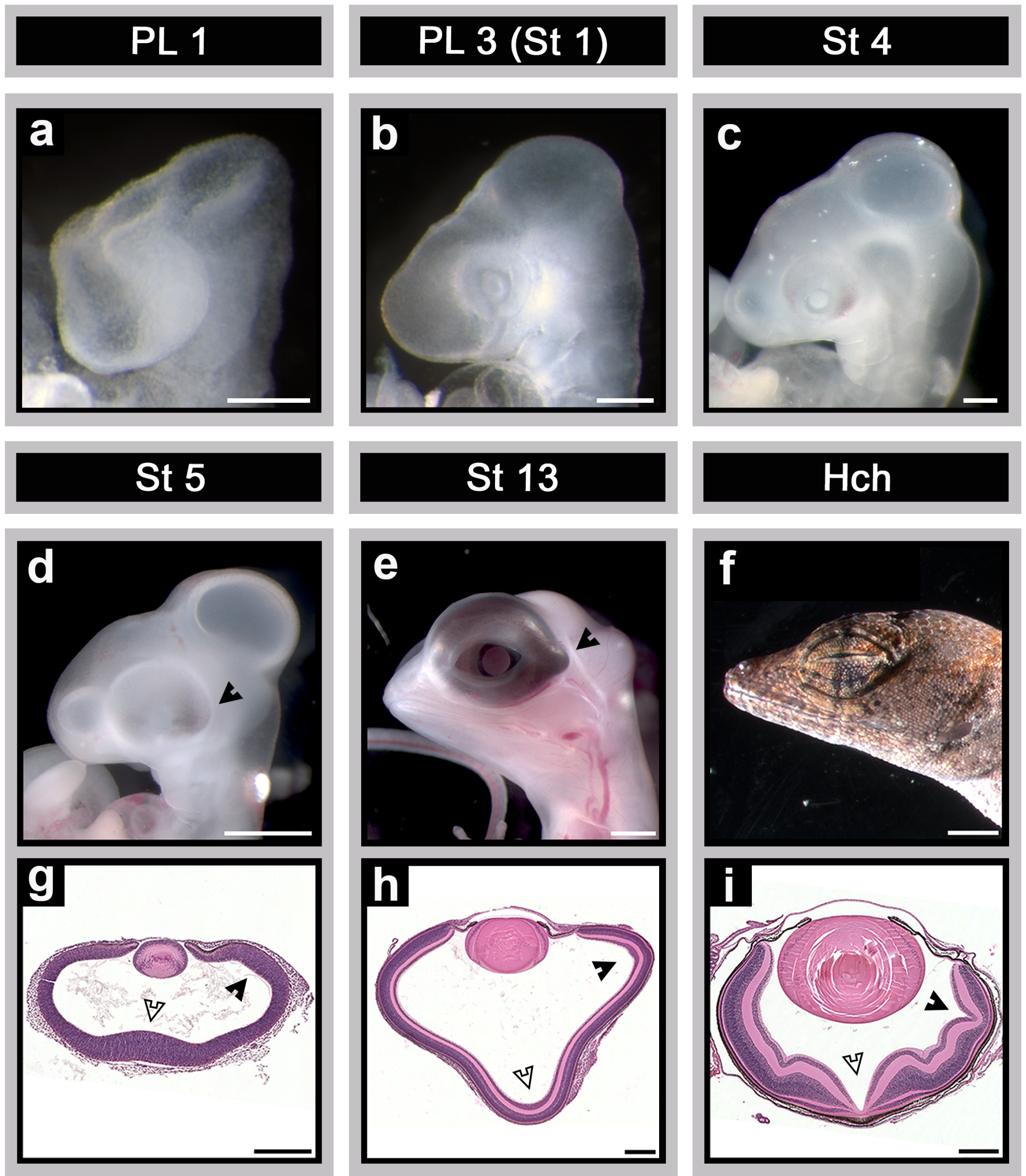


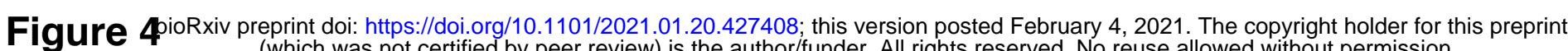
(which was not certified by peer review) is the author/funder. All rights reserved. No reuse allowed without permission

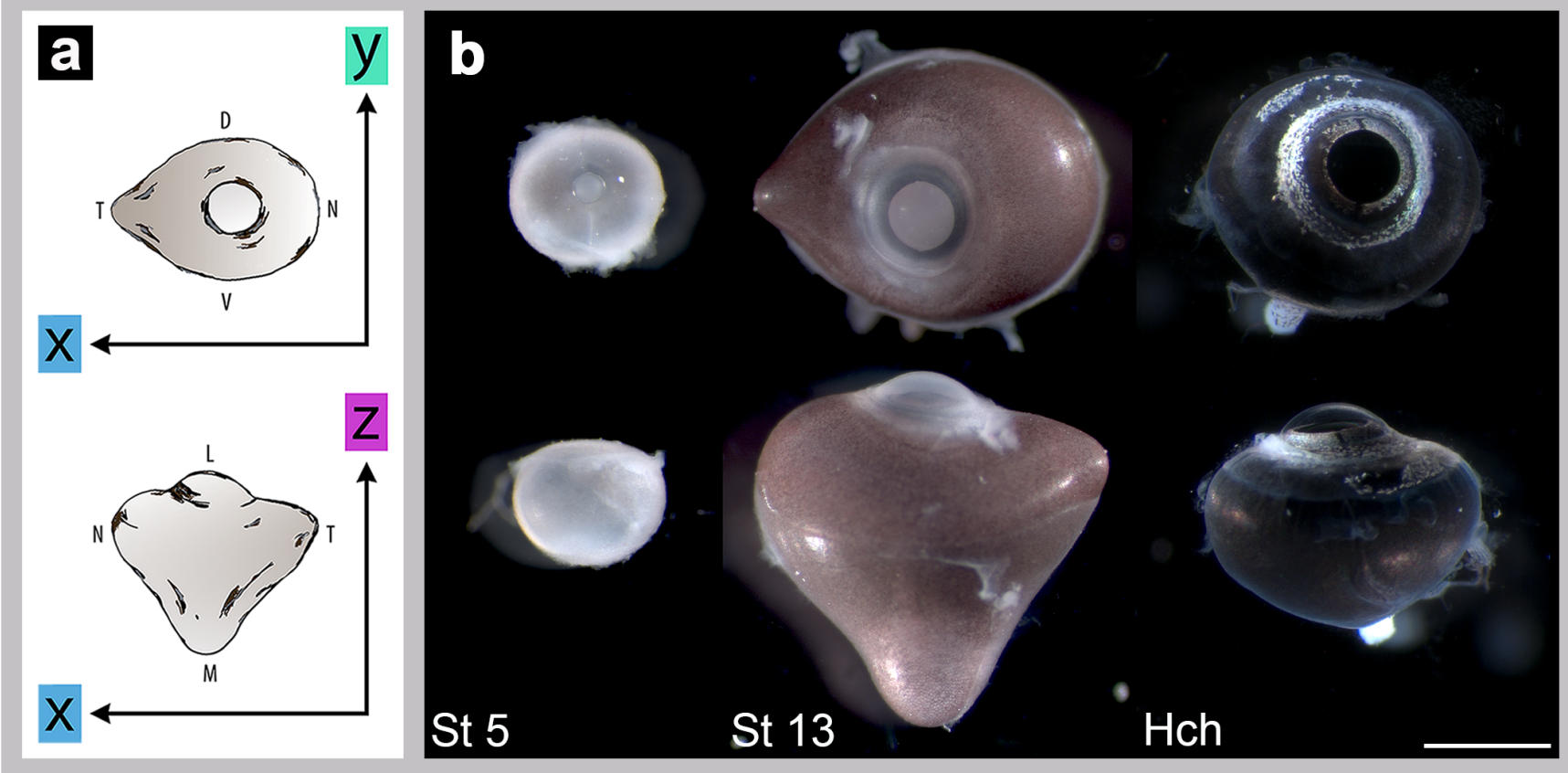

\section{$\mathbf{G}$}
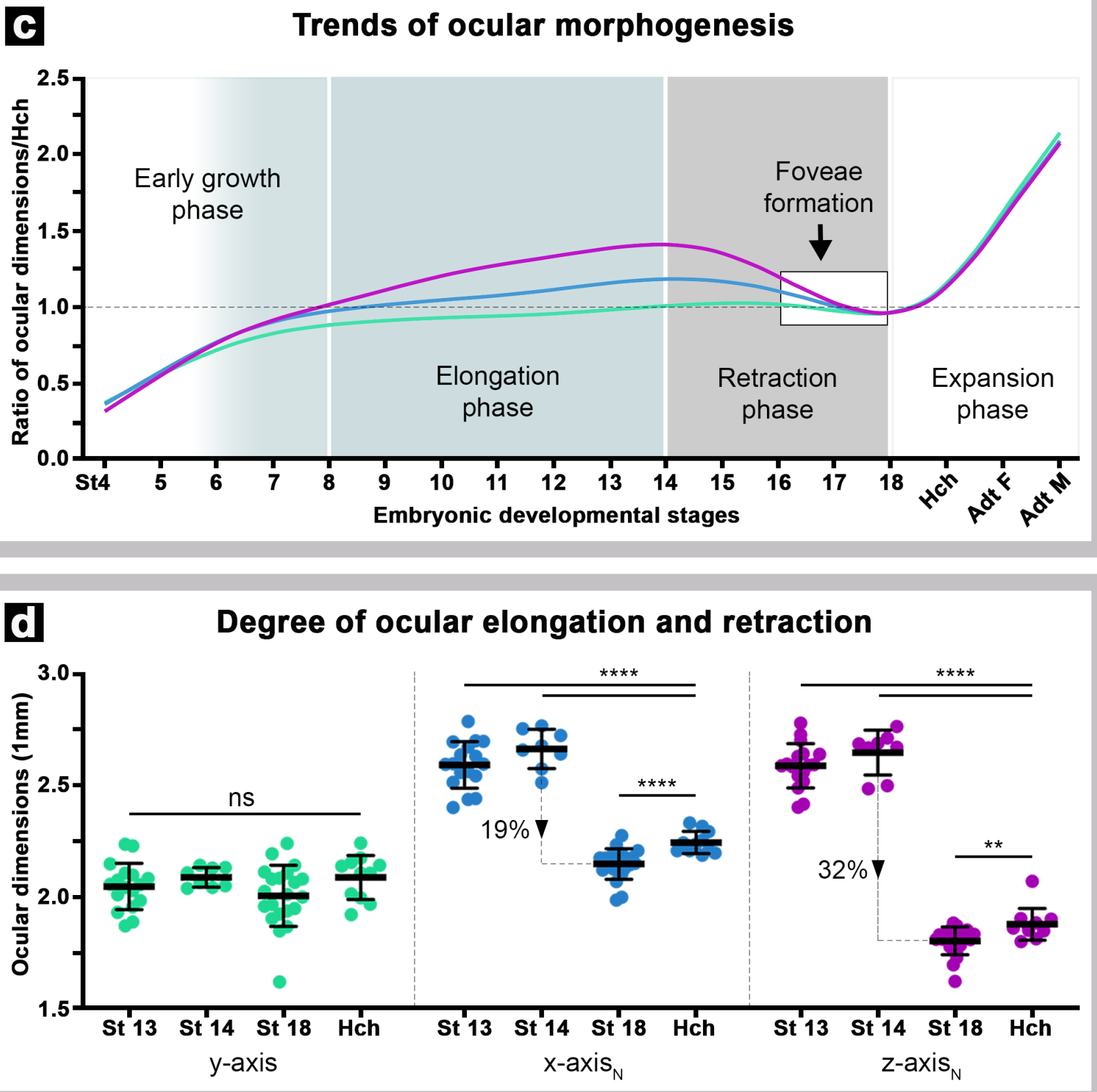
bioRxiv preprint doi: https://doi.org/10.1101/2021.01.20.427408; this version posted February 4, 2021. The copyright holder for this preprint 5 (which was not certified by peer review) is the author/funder. All rights reserved. No reuse allowed without permission.

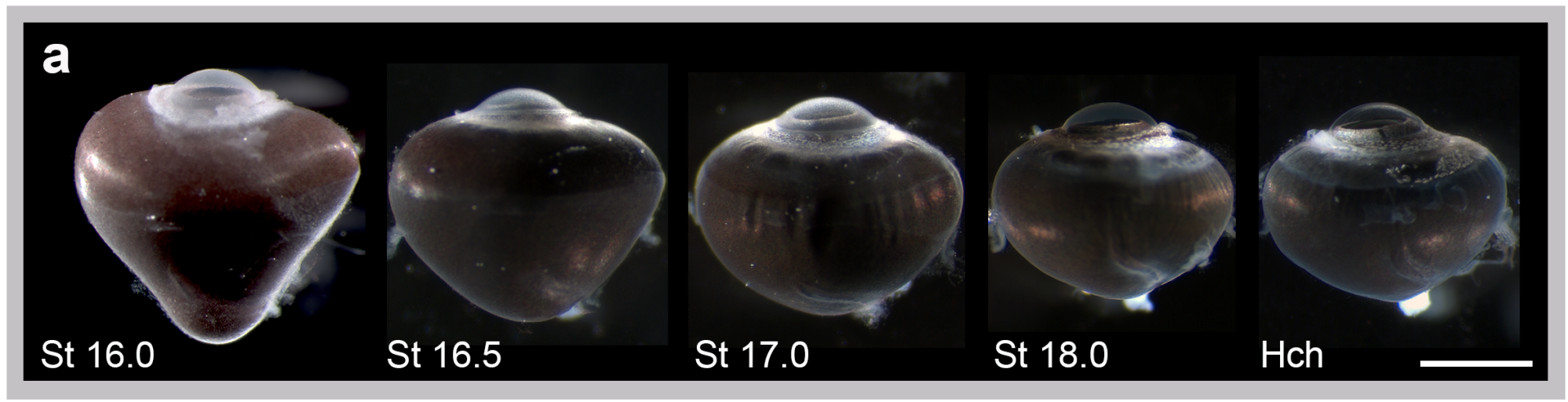

0

\section{4}

St 17.0

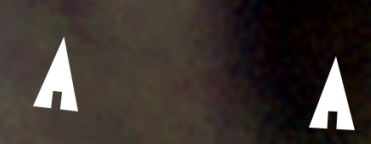

$\Lambda$

\section{Exterior ocular surface (smooth vs wrinkled)}

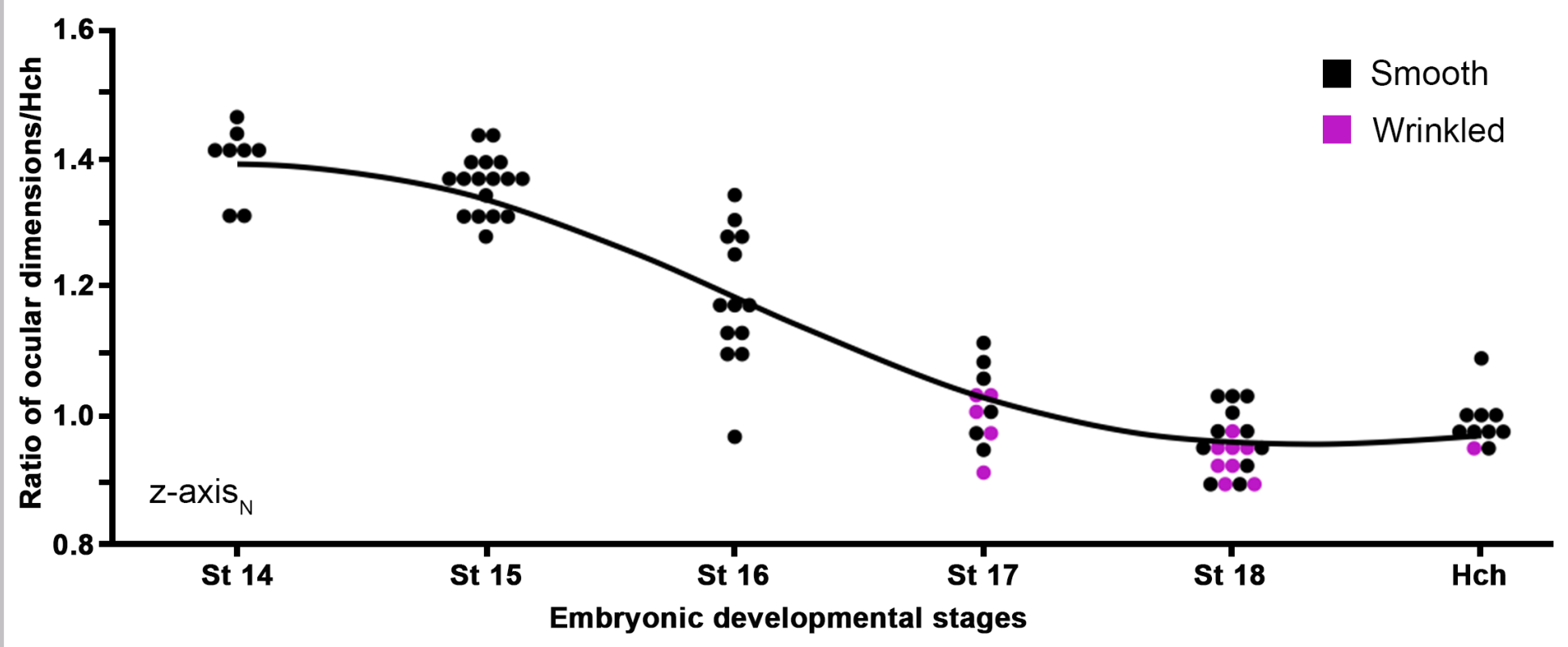




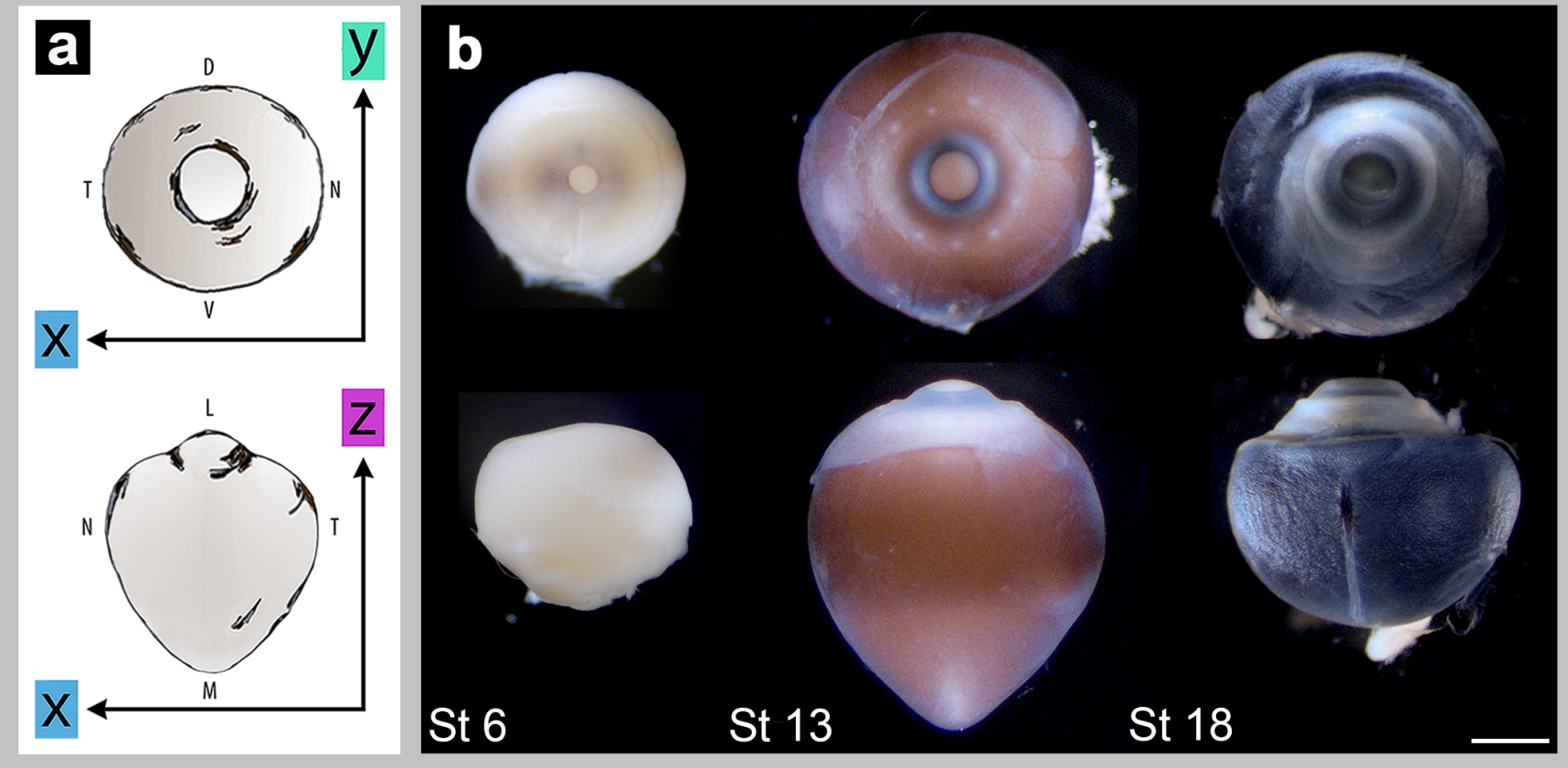

\section{G}
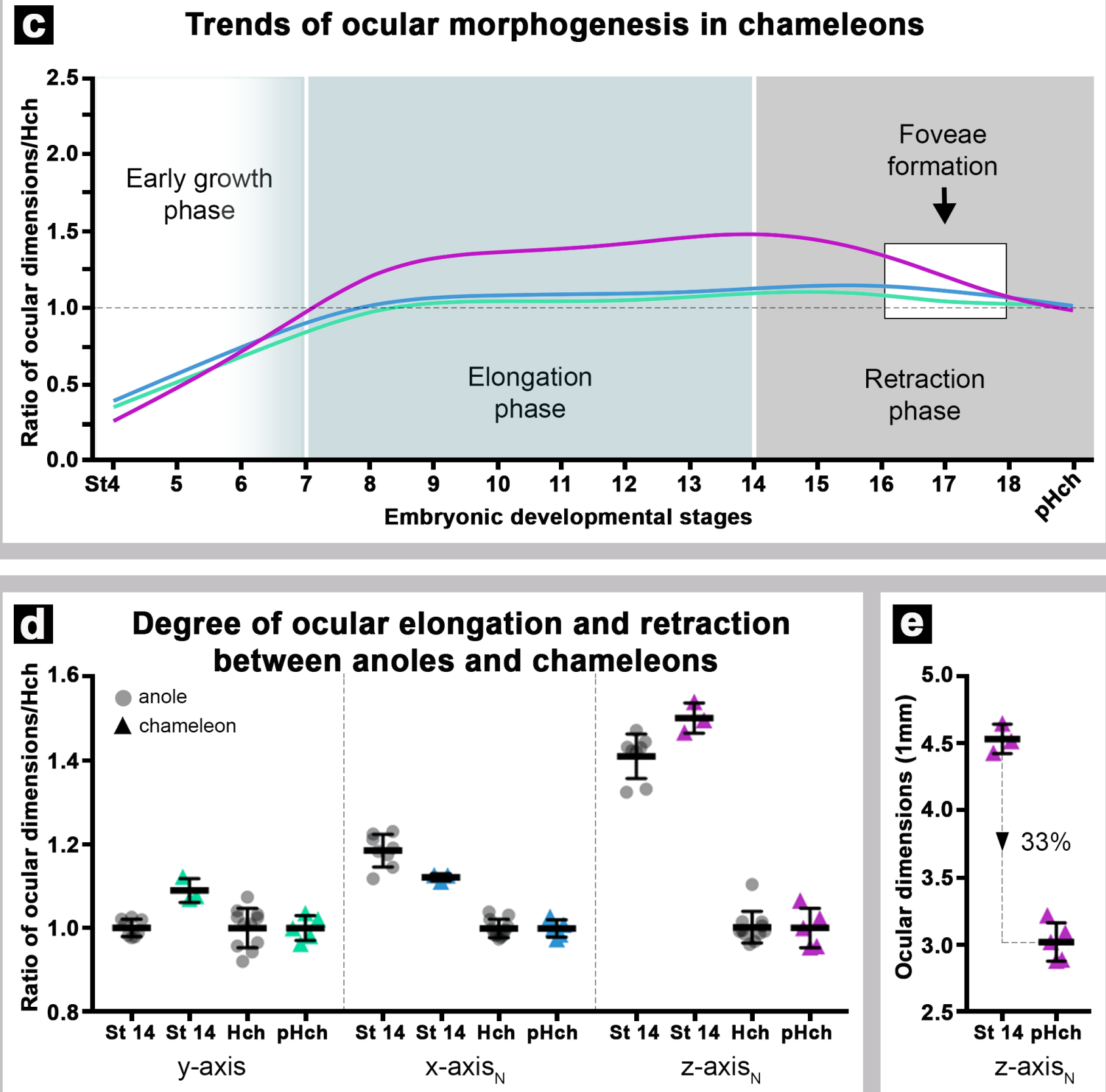